University of Wollongong

Research Online

Australian Institute for Innovative Materials -

Papers

Australian Institute for Innovative Materials

$1-1-2018$

Synergistically Enhanced Interfacial Interaction to Polysulfide via N,O DualDoped Highly Porous Carbon Microrods for Advanced Lithium-Sulfur Batteries

Nana Wang

University of Wollongong, Taiyuan University of Technology, nw415@uowmail.edu.au

Zhongfei $\mathrm{Xu}$

University of Wollongong, xuzfei@buaa.edu.cn

Xun Xu

University of Wollongong, xun@uow.edu.au

Ting Liao

Queensland University of Technology, tingl@uow.edu.au

Bin Tang

Taiyuan University of Technology

See next page for additional authors

Follow this and additional works at: https://ro.uow.edu.au/aiimpapers

Part of the Engineering Commons, and the Physical Sciences and Mathematics Commons

Research Online is the open access institutional repository for the University of Wollongong. For further information contact the UOW Library: research-pubs@uow.edu.au 


\title{
Synergistically Enhanced Interfacial Interaction to Polysulfide via N,O Dual-Doped Highly Porous Carbon Microrods for Advanced Lithium-Sulfur Batteries
}

\author{
Abstract \\ Lithium-sulfur (Li-S) batteries have received tremendous attention because of their extremely high \\ theoretical capacity (1672 mA h g -1) and energy density (2600 W h kg -1). Nevertheless, the \\ commercialization of Li-S batteries has been blocked by the shuttle effect of lithium polysulfide \\ intermediates, the insulating nature of sulfur, and the volume expansion during cycling. Here, hierarchical \\ porous $\mathrm{N}, \mathrm{O}$ dual-doped carbon microrods (NOCMs) were developed as sulfur host materials with a large \\ pore volume $(1.5 \mathrm{~cm} 3 \mathrm{~g}-1)$ and a high surface area $(1147 \mathrm{~m} 2 \mathrm{~g} \mathrm{-1})$. The highly porous structure of the \\ NOCMs can act as a physical barrier to lithium polysulfides, while $\mathrm{N}$ and $\mathrm{O}$ functional groups enhance the \\ interfacial interaction to trap lithium polysulfides, permitting a high loading amount of sulfur (79-90 wt \% \\ in the composite). Benefiting from the physical and chemical anchoring effect to prevent shuttling of \\ polysulfides, S@NOCMs composites successfully solve the problems of low sulfur utilization and fast \\ capacity fade and exhibit a stable reversible capacity of $1071 \mathrm{~mA} \mathrm{~h} \mathrm{~g}-1$ after 160 cycles with nearly $100 \%$ \\ Coulombic efficiency at $0.2 \mathrm{C}$. The $\mathrm{N}, \mathrm{O}$ dual doping treatment to porous carbon microrods paves a way \\ toward rational design of high-performance Li-S cathodes with high energy density. \\ Disciplines \\ Engineering | Physical Sciences and Mathematics

\section{Publication Details} \\ Wang, N., Xu, Z., Xu, X., Liao, T., Tang, B., Bai, Z. \& Dou, S. (2018). Synergistically Enhanced Interfacial \\ Interaction to Polysulfide via N,O Dual-Doped Highly Porous Carbon Microrods for Advanced Lithium- \\ Sulfur Batteries. ACS Applied Materials and Interfaces, 10 (16), 13573-13580.

\section{Authors} \\ Nana Wang, Zhongfei Xu, Xun Xu, Ting Liao, Bin Tang, Zhongchao Bai, and Shi Xue Dou
}

This journal article is available at Research Online: https://ro.uow.edu.au/aiimpapers/3090 


\section{Synergistically Enhanced Interfacial Interaction to}

\section{Polysulfide via N,O Dual-Doped Highly Porous}

\section{Carbon Microrods for Advanced Lithium-Sulfur}

\section{Batteries}

Nana Wang, ${ }^{a b}$ Zhongfei Xu, ${ }^{a}$ Xun Xu, ${ }^{a}$ Ting Liao, ${ }^{c}$ Bin Tang, ${ }^{b}$ Zhongchao Bai, ${ }^{a b}$ and Shixue $\operatorname{Dou}^{*^{a}}$

${ }^{a}$ Institute for Superconducting and Electronic Materials, University of Wollongong, Innovation Campus, Squires Way, New South Wales 2500, Australia

${ }^{\mathrm{b}}$ College of Materials Science and Engineering, Taiyuan University of Technology, Taiyuan, Shanxi 030024, P. R. China.

${ }^{\mathrm{c}}$ School of Chemistry, Physics and Mechanical Engineering, Queensland University of Technology, QLD 4000, Australia,

Keywords: nitrogen and oxygen; dual doped; lithium-sulfur batteries; highly porous; carbon. 
Abstract: Lithium-sulfur (Li-S) batteries have received tremendous attention due to their extremely high theoretical capacity (1672 $\mathrm{mAh} \mathrm{g}^{-1}$ ) and energy density (2600 Wh kg-1). Nevertheless, the commercialization of Li-S batteries has been blocked by the shuttle effect of lithium polysulfide intermediates, the insulating nature of sulfur, and the volume expansion during cycling. Here, hierarchical porous N, O dual doped carbon microrods (NOCMs) were developed as sulfur host materials with large pore volume $\left(1.5 \mathrm{~cm}^{3} \mathrm{~g}^{-1}\right)$ and high surface area $\left(1147 \mathrm{~m}^{2} \mathrm{~g}^{-1}\right)$. The highly porous structure of the NOCMs can act as a physical barrier to lithium polysulfides, while $\mathrm{N}$, O functional groups enhance the interfacial interaction to trap lithium polysulfides, permitting an high loading amount of sulfur (79-90 wt\% in the composite). Benefiting from the physical and chemical anchoring effect to prevent shuttling of polysulfides, S@NOCMs composites successfully solve the problems of low sulfur utilization and fast capacity fade, and exhibit a stable reversible capacity of $1071 \mathrm{mAh} \mathrm{g}^{-1}$ after 160 cycles with nearly $100 \%$ Coulombic efficiency at $0.2 \mathrm{C}$. The N, O dual doping treatment to porous carbon microrods pave a way towards rational design of high-performance Li-S cathodes with high energy density. 


\section{Introduction}

In the face of severe energy shortages and environmental pollution, adopting renewable sources (e.g. solar and wind), and hybrid or pure electric vehicles are the best solutions and have promoted the rapid development of rechargeable battery systems. Commercial Li-ion batteries (LIBs), which have dominated the rechargeable-battery market in the past few decades, are unable to meet the high-energy density demands of those devices due to the low theoretical capacity of traditional cathode materials. In this respect, Li-S batteries are regarded as one of the most promising next-generation secondary batteries due to their high theoretical capacity (1672 $\mathrm{mAh} \mathrm{g}^{-1}$ ) and energy density (2600 $\left.\mathrm{Wh} \mathrm{kg}^{-1}\right)$, which is several orders of magnitude higher than for traditional LIB cathode materials. ${ }^{1-4}$ Elemental sulfur is particularly cheap and abundant in the Earth's crust, and thus would be beneficial for large-scale energy storage. Despite these significant advantages, the practical application of Li-S batteries still faces some unavoidable problems. First, the insulating properties of sulfur leads to limited diffusion of lithium ions and electrons, as well as low utilization of the active sulfur mass. Second, the volume change caused by the stepwise reaction of sulfur to form $\mathrm{Li}_{2} \mathrm{~S}$ during cycling is about $80 \%$, resulting in structural changes and separation of the current collector from the sulfur electrode. Furthermore, the lithium polysulfides (the discharge intermediate $\mathrm{Li}_{2} \mathrm{~S}_{x}$ products) are soluble in the liquid electrolyte and could migrate between the electrodes, which is known as the shuttle effect and is the key factor behind the poor cycling stability, low Coulombic efficiency, and self-discharge..$^{5-7}$

Many efforts have been devoted to overcoming the shuttle effect by trapping polysulfides in the sulfur cathode. Various types of additives such as polar polymer additives, ${ }^{8}$ polar metal oxides/sulfides $\left(\mathrm{MnO}_{2},{ }^{9} \mathrm{TiS}_{2}{ }^{10}\right)$, and metal-organic frameworks ${ }^{11}$ have been employed as sulfur 
host materials that work via chemical or physical confinement to achieve improved cycling properties. Among them, carbonaceous materials are the most frequently used sulfur hosts due to their very light weight, high electrical conductivity, and low cost. ${ }^{12-17}$ In particular, the porous structured carbons, which could physically confine lithium polysulfide species and avoid their leaching during cycling, have attracted considerable attention. ${ }^{18}$ Pure carbon is a substance without polarity, however, and hence, it possesses a limited ability to anchor sulfur and only has weak chemical binding with polar lithium polysulfides, decreasing the adsorption and trapping effect. $^{7,19}$ Based on this background, heteroatom doped carbon materials are a better choice because they can combine the advantages of lightweight carbon and strong anchoring at heteroatom sites, which strengthens the physical confinement of and chemical affinity for sulfur as well as lithium polysulfides. This has opened up a new way to reduce the shuttle effect, increase the sulfur utilization, and provide interfaces for lithium sulfide deposition. ${ }^{20}$ For example, Zhang et al. were the first to use graphene oxide to immobilize $\mathrm{S}$, generating high reversible capacities of 950-1400 $\mathrm{mA} \mathrm{h} \mathrm{g}^{-1} \cdot{ }^{21}$ After years of effort, various strong anchoring sites, such as on nitrogen-doped carbon, ${ }^{22}$ nitrogen/sulfur co-doped graphene sponge, ${ }^{23}$ boron/nitrogen dual-doped carbon layers, ${ }^{24}$ etc., were reported to relieve the shuttle effect of polysulfides via chemical binding. Among them, $\mathrm{N}$ and $\mathrm{O}$, or even co-doping, are better options to suppress the shuttle effect, as indicated by calculations. ${ }^{20}$ Consequently, designing a porous carbon with suitable pore size, pore volume, and surface area in terms of proper chemical heteroatom doping is expected to achieve higher sulfur loading and facilitate a better anchoring of the polysulfides.

In view of the above discussion, hierarchical porous $\mathrm{N}$, $\mathrm{O}$ dual doped carbon microrods (NOCMs) with a highly porous, graphitic-crystalline matrix and enriched N/O groups were prepared through a novel approach, using $\mathrm{Ni}(\mathrm{DMG})_{2}(\mathrm{DMG}=$ dimethylglyoxime $)$ as the 
precursor, followed by carbonization and acid etching, to serve as sulfur hosts for Li-S batteries in liquid-based electrolytes. NOCMs exhibit a large surface area of $1147 \mathrm{~m}^{2} \mathrm{~g}^{-1}$ and pore volume of $1.5 \mathrm{~cm}^{3} \mathrm{~g}^{-1}$, which can not only provide enough space to encapsulate sulfur or lithium polysulfides during charge/discharge processes, but also offers faster electrolyte access to the active sulfur materials. In addition, N, O dual doped carbon microrods could provide high electrical conductivity and decrease the ion diffusion length, facilitating fast electron/ion transfer. Most importantly, the nitrogen and oxygen doping strengthens the chemical binding between the carbon and the lithium polysulfides, relieving the shuttle effect to improve the electrochemical performance. Both the physical confinement and the chemical binding of polysulfides on NOCM materials lead to the high specific capacity of $1071 \mathrm{mAh} \mathrm{g}^{-1}$ at the $0.2 \mathrm{C}$ rate. Even at high current density of 4 C, S@NOCM material can still maintain 558 mAh g-1.

\section{Experimental Section}

Material Synthesis: All reagents were used without any further purification after purchase. First, $10 \mathrm{mmol}$ DMG was dissolved in $100 \mathrm{~mL}$ of ethyl alcohol. Then, $100 \mathrm{~mL}$ of $0.01 \mathrm{M}$ $\mathrm{NiCl}_{2} \cdot 6 \mathrm{H}_{2} \mathrm{O}$ was added dropwise to the above solution under vigorous stirring. The resulting $\mathrm{Ni}(\mathrm{DMG})_{2}$ precipitate was filtered and washed with distilled water and alcohol several times. Then, the products were calcined at $400{ }^{\circ} \mathrm{C}$ for $2 \mathrm{~h}$ and subsequently at $800{ }^{\circ} \mathrm{C}$ for $10 \mathrm{~h}$ under argon atmosphere with a heating rate of $2{ }^{\circ} \mathrm{C} \min ^{-1}$. The carbonized products were treated with a mixed solution consisting of $10 \mathrm{~mL} \mathrm{H}_{2} \mathrm{O}_{2}$ (30\%), $10 \mathrm{~mL}$ concentrated $\mathrm{HCl}$, and $20 \mathrm{~mL}$ distilled water at $120{ }^{\circ} \mathrm{C}$ for $4 \mathrm{~h}$ to remove the Ni metal particles. Finally, the NOCM product was obtained after washing three times with water.

Synthesis of the S@NOCMs composite: Typically, the appropriate amount of sulfur powder and NOCMs were mixed together and put into a sealed container filled with argon atmosphere, 
before heating at $155^{\circ} \mathrm{C}$ for $12 \mathrm{~h}$. The obtained S@NOCMs composite was heated under flowing Ar atmosphere at $200{ }^{\circ} \mathrm{C}$ for 30 min to remove the residual sulfur from the surfaces of the S@NOCMs.

Materials Characterization: X-ray powder diffraction (XRD) patterns of the products were collected using a Bruker D8 Advance X-ray diffractometer $(\mathrm{Cu} \mathrm{K} \alpha$ radiation, $\lambda=1.5418 \AA$, Germany). The microstructure and morphology of samples were characterized by transmission electron microscope (TEM, JEOL JEM 1011, Japan), field-emission scanning electron microscope (SEM, SUPRA ${ }^{\mathrm{TM}}$ 55, Germany), and high-resolution transmission electron microscope (HRTEM, JEOL2100, Japan). To measure the sulfur loadings in the composite, thermogravimetric analysis (TGA) was carried out on a thermal analyzer (Mettler Toledo TGA/SDTA851, Switzerland) in flowing nitrogen gas. Nitrogen adsorption/desorption isotherms were characterized on a specific analyzer at 77 K (ASAP 2020HD88, Micromeritics, USA). Xray photoelectron spectroscopy (XPS) was applied to analyze the chemical valences of samples (ESCALAB 250, USA).

Electrochemical Measurements: To make the working electrodes, $75 \mathrm{wt} \%$ active materials, $15 \mathrm{wt} \%$ of the conductive agent carbon black, and $10 \mathrm{wt} \%$ polyvinylidene difluoride (PVDF) were mixed together with a few drops of N-methylpyrrolidone (NMP) to form a homogeneous slurry. The as-prepared slurry was spread on cleaned aluminum foil and then punched into discs with a diameter of $12 \mathrm{~mm}$. The areal sulfur mass loading could be controlled by monitoring the surface coating thickness and slurry concentration. The 2032-coin cells were assembled in an argon-filled glove box (Mikrouna, Super 1220/750/900), with metallic lithium as the anode and Celgard 2400 as the separator. The electrolyte was $1 \mathrm{M}$ bis(trifluoromethane)sulfonamide lithium salt dissolved in 1,3-dioxolane and dimethyl ether (DME) (1:1 v/v) containing $1 \mathrm{wt} \% \mathrm{LiNO}_{3}$. 
The amount of electrolyte used in each coin cell depends on the sulfur loading mass, and the electrolyte/sulphur ratio of the cells is $20 \mu \mathrm{L} \mathrm{mg}$. The galvanostatic charge/discharge performance was investigated on Land-CT2001A battery cyclers at $25{ }^{\circ} \mathrm{C}$ with the voltage window between 1.4 and $2.8 \mathrm{~V}$. Cyclic voltammetry (CV) profiles were collected on electrochemical workstations (LK2005A, China) at a scan rate of $0.1 \mathrm{mV} \mathrm{s}^{-1}$. Electrochemical impedance spectra were collected using an electrochemical workstation (AUTOLAB PGSTAT302) in the frequency range of $100 \mathrm{kHz}$ to $0.01 \mathrm{~Hz}$.

Computational method: All density functional theory (DFT) calculations were performed using the Vienna Ab Initio Simulation Package (VASP). The projector augmented wave (PAW), pseudopotential and generalized gradient approximation (GGA) were applied to treat the exchange correlation energy with the Perdew-Burke-Ernzerhof (PBE) functional. The cut-off energy for the plane wave basis is $550 \mathrm{eV}$, and k-point sampling was restricted to the Gamma point only. Equilibrium geometries were obtained by the minimum energy principle until the force converged to $0.02 \mathrm{eV} / \AA$. All structures in the calculations were relaxed until the convergence tolerance for the force on each atom was smaller than $0.02 \mathrm{eV}$. The energy convergence criterion was set to be $1 \times 10^{-4} \mathrm{eV}$ for self-consistent calculations. For the simulation, a piece of graphene was cut with the dangling bonds saturated by $\mathrm{H}$ atoms and put in the middle of a lattice box with $a=b=c=30 \AA$, which is large enough to avoid unexpected interactions between atoms in different supercells. The adsorption energy $\left(E_{\mathrm{ad}}\right)$ is calculated as follows (1):

$E_{\text {ad }}=E_{\text {molecule }}+E_{\text {substrate }}-E_{\text {tot }}$

where $E_{\text {total, }}, E_{\text {molecule, }}$ and $E_{\text {substrate }}$ refer to the energy of whole system, of the molecule $\left(\mathrm{Li}_{2} \mathrm{~S}_{4}\right)$, and of the substrate (carbon, N, O co-doped carbon), respectively. 


\section{Results and discussion}

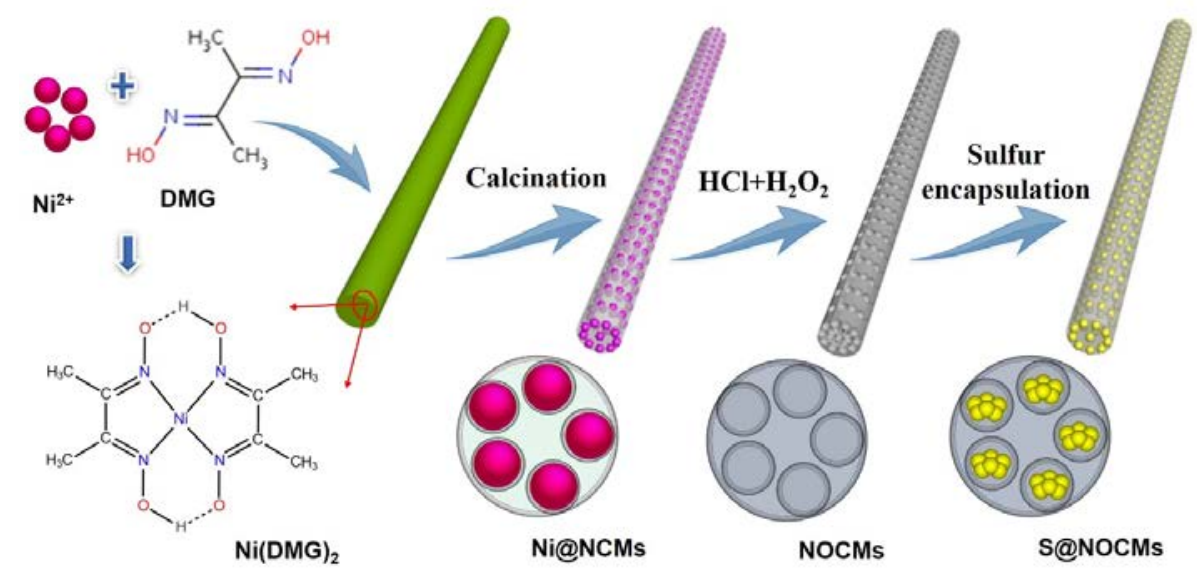

Figure 1. Schematic illustration of the formation process for S@NOCMs composite.

The facile route to synthesize S@NOCMs composite is illustrated in Figure 1. First, uniform $\mathrm{Ni}(\mathrm{DMG})_{2}$ microrods were prepared by a simple complexation reaction of $\mathrm{NiCl}_{2} \cdot 6 \mathrm{H}_{2} \mathrm{O}$ and DMG (Figure S1 in the Supporting Information). ${ }^{25}$ Then, the Ni(DMG) 2 microrods were annealed in $\mathrm{Ar}$ atmosphere $\left(800{ }^{\circ} \mathrm{C}\right)$ to produce Ni particles encapsulated in $\mathrm{N}$-doped carbon microrods (Ni@NCMs). Because of their flexible structure, Ni@NCMs maintained the basically original one-dimensional (1D) morphology of the $\mathrm{Ni}(\mathrm{DMG})_{2}$ microrods (Figure S2). The nitrogen groups were successfully introduced into the carbon material by an in-situ reaction through carbonization of DMG with enrichment of the nitrogen element. In the meantime, the nickel ions were reduced by the carbon material into nickel metal nanoparticles, which were evenly distributed in the carbon material to act as in-situ templates (Figure S2c). After treatment by a mixture of hydrochloric acid and hydrogen peroxide, the Ni nanoparticles were removed and highly porous nitrogen and oxygen co-doped carbon material was obtained, because the carbon surface was oxidized by the mixed acids, and many functional groups were introduced in forms of carboxyl, carbonyl, hydroxyl, etc. This kind of N, O-doped carbon material has many 
advantages when used as a host material for sulfur. First, its highly porous structure can supply electrolyte access to the electrode and act as a reactor for lithium and sulfur. Second, the nitrogen doping can improve the conductivity of the composite and alleviate the shuttle effect of lithium polysulfides. ${ }^{26-28}$ Sulfur was embedded in the porous structure using a melt diffusion strategy. The sulfur on the outer surface of the carbon would be removed by evaporation.
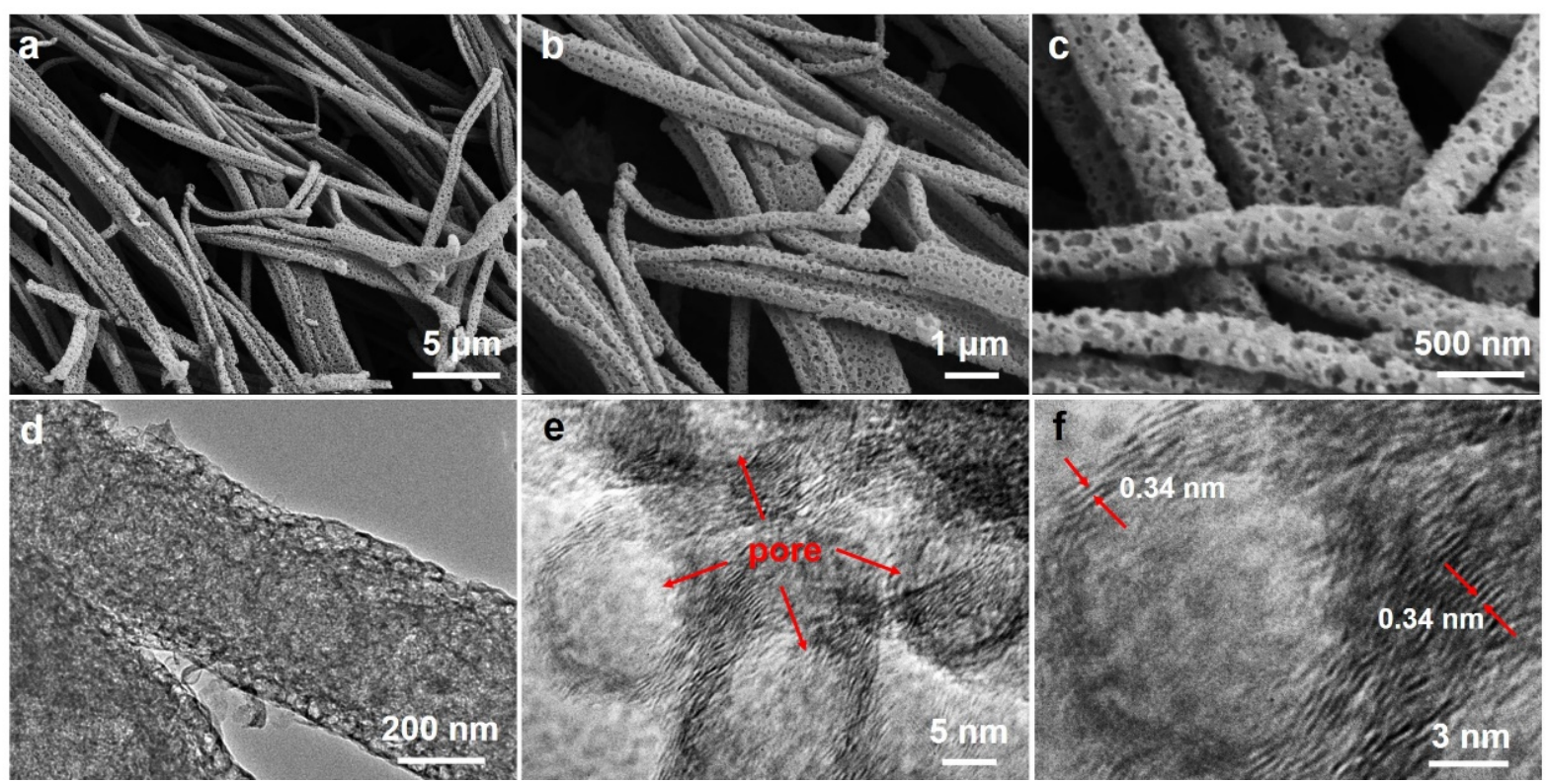

Figure 2. (a - c) SEM images, (d) TEM image, and (e, f) HRTEM images of NOCMs sample.

Figure 2 shows SEM, TEM, and HRTEM images of the synthesized samples. As shown in Figure 2a, the NOCMs sample is made up of uniform one-dimensional microrods with diameters ranging from 300 to $500 \mathrm{~nm}$ and lengths reaching dozens of micrometers. Close inspection shows that the surface of the NOCMs material has plentiful mesopores or even some macropores due to the removal of Ni particles (Figure 2b, c, Figure S3). The contrast in the TEM image (Figure 2d), which is characteristic of porous structure, is well supported by the SEM observations. The HRTEM images display some hollow sphere-like structures on the microrods, which are inherited from the Ni nanoparticles (Figure 2e). This hollow structure is beneficial for 
trapping sulfur and its polysulfides and leads to better electrochemical properties. Because of the catalytic effect of nickel, the carbon material is short-range ordered, and the interlayer spacing, $d$, is $0.34 \mathrm{~nm}$ (Figure 2f). The Raman spectrum of NOCMs contains a D (disordered carbon) band at $1324 \mathrm{~cm}^{-1}$ and $\mathrm{G}$ (graphitic carbon) band at $1580 \mathrm{~cm}^{-1}$ (Figure S4). The intensity of the D band is a little stronger than that of the G band, indicating that the N, O-dual doping and the edge defects have caused structural distortion, which is often observed in heteroatoms doped carbon materials. $^{29,30}$
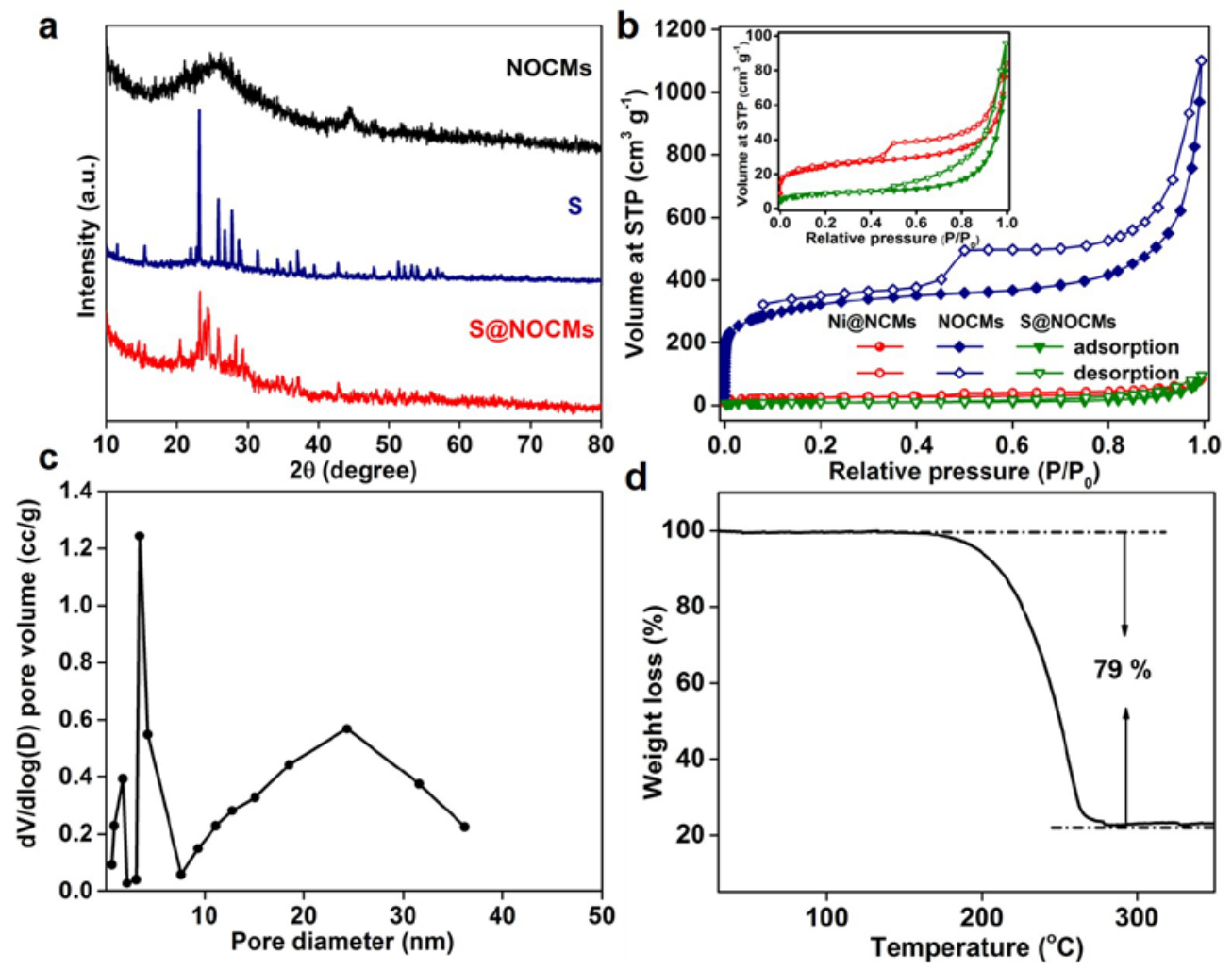

Figure 3. (a) XRD patterns of NOCMs, sulfur powder, and S@NOCMs (79 wt\% S) composite. (b) Nitrogen adsorption/desorption isotherms of Ni@NCMs, NOCMs, and S@NOCMs (79 wt\% S) composite, with the inset showing an enlargement of the isotherms for NOCMs and S@NOCMs. (c) Corresponding pore size distribution of NOCMs sample. (d) TGA curve of S@NOCMs composite. 
The composition and porous properties of NOCMs, as well as S@NOCMs composite, were investigated by XRD and Brunauer-Emmett-Teller (BET) analysis. The X-ray diffraction pattern of NOCMs exhibits one broad peak centered at $23^{\circ}$, corresponding to the (002) plane, while a small peak at $44^{\circ}$ could be indexed to (100) plane, indicating the graphitized structure in NOCMs. ${ }^{29,30}$ This is in line with the HRTEM images and favorable for the conductivity of the composite. The sharp peaks shown in Figure 3a could be attributed to the fddd orthorhombic structure of crystalline sulfur. ${ }^{31}$ Even when a high amount of sulfur is loaded on NOCMs, however, the characteristic peaks of sulfur show much weaker intensity compared to the original crystalline sulfur, which demonstrates that the elemental sulfur mainly penetrates the pores of the NOCMs. ${ }^{29,32}$ This result is further confirmed by BET examination (Figure 3b, 3c and Figure S5). NOCMs exhibits a very high surface area of $1147 \mathrm{~m}^{2} \mathrm{~g}^{-1}$ and high pore volume of $1.5 \mathrm{~cm}^{3} \mathrm{~g}^{-1}$ with Type IV isoherms and Type H3 hysteresis loops. ${ }^{33}$ After loading with sulfur, however, the surface area and pore volume rapidly decreased to $31 \mathrm{~m}^{2} \mathrm{~g}^{-1}$ and $0.14 \mathrm{~cm}^{3} \mathrm{~g}^{-1}$, respectively, suggesting that the sulfur had filled in the pores of NOCMs. The S@NOCMs composites displayed an adjustable high sulfur content of $79 \mathrm{wt} \%$ and $90 \mathrm{wt} \%$, as determined by TGA analysis (Figure 3b and Figure S6). These features could be ascribed to the simultaneous chemical and physical anchoring effect for sulfur or lithium polysulfides on the N, O-dual doped micro/mesoporous carbon microrods. 

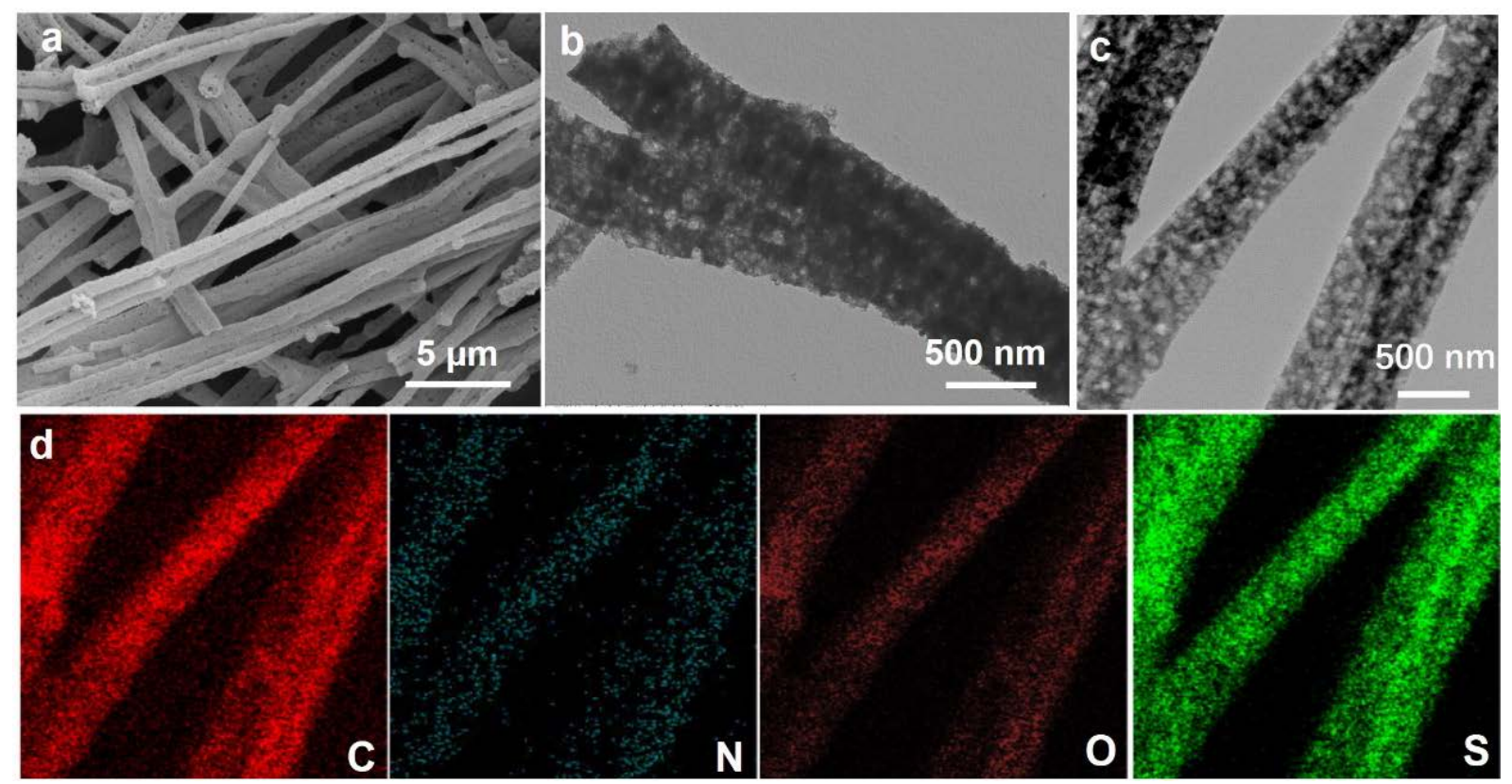

Figure 4. (a) SEM image and (b) TEM image of S@NOCMs composite. (c) STEM image and (d) corresponding carbon, nitrogen, oxygen, and sulfur elemental mappings of S@NOCMs.

SEM and TEM images of S@NOCMs composite with 79 wt\% sulfur loading are displayed in Figure 4a-c. The overview of the composite via SEM presents a one-dimensional structure very similar to that of NOCMs (Figure 4a). The TEM image of S@NOCMs composite (Figure 4b) is darker than that of NOCMs (Figure 2d) because of the loading of sulfur. The scanning TEM (STEM) image, however, still displays a porous-like structure with obviously contrast, which makes it clear that the sulfur was homogeneously distributed on the carbon framework and had infiltrated into the mesopores. In Figure 4d, energy dispersive X-ray spectroscopy (EDS) reveals the presence of $\mathrm{C}, \mathrm{O}, \mathrm{N}$, and $\mathrm{S}$ elements in the S@NOCMs composite, indicating the successfully incorporation of $\mathrm{N}$ and $\mathrm{O}$ atoms into the porous carbon microrods and the homogeneous distribution of S. 

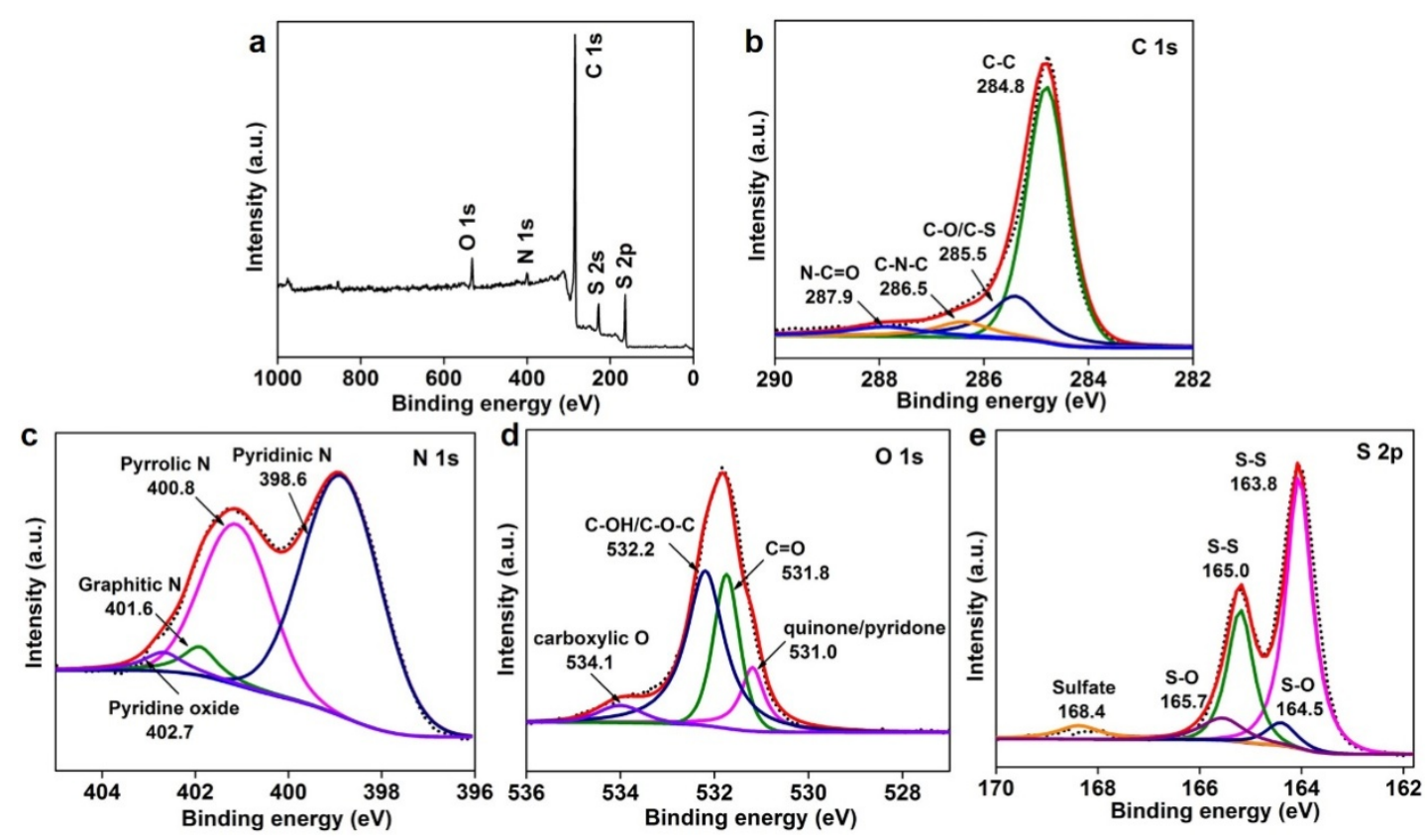

Figure 5. (a) XPS survey spectrum of S@NOCMs composite. High resolution XPS spectra of (b) C 1s, (c) N 1s, (d) O 1s, and (e) S 2p.

X-ray photoelectron spectroscopy (XPS) was conducted to further investigate the elemental composition and functional groups of the S@NOCMs composites, and the results are shown in Figure 5. The survey spectrum clearly demonstrates the presence of the $\mathrm{C}, \mathrm{N}, \mathrm{O}$, and $\mathrm{S}$ elements in the S@NOCMs composite (Figure 5a). The C 1s spectrum displays a major peak at $284.8 \mathrm{eV}$, which could be attributed to $s p^{2}$ hybridized carbon, and the other three weak peaks at 285.5, 286.5, and $287.9 \mathrm{eV}$ can be ascribed to C-O/C-S, C-N-C, and N-C=O species, respectively. ${ }^{31}$ In the $\mathrm{N}$ 1s spectrum, four peaks at 398.6, 400.8, 401.6, and $402.7 \mathrm{eV}$ are detected and, in turn, can be assigned to pyridinic $\mathrm{N}$, pyrrolic $\mathrm{N}$, graphitic $\mathrm{N}$, and pyridine oxide $\mathrm{N}$. The peak area ratio of pyridinic and pyrrolic $\mathrm{N}$ to total $\mathrm{N}$ is as high as $93 \%$, permitting a more effective chemical anchoring effect. ${ }^{34}$ Because the graphitic $\mathrm{N}$ provides its $p$ electrons to form a $\pi$-conjugated system, improving the charge density in the doping region, it thus possesses no additional lone pair electrons to anchor lithium sulfide through $\mathrm{Li}_{2} \mathrm{~S}_{x}-\mathrm{N}$ chemical bonding. ${ }^{20}$ Similarly, the doped 
$\mathrm{O}$ atoms play an analogous role to $\mathrm{N}$ in the S@NOCMs composite. In Figure 5d, the binding energy peaks observed in the $\mathrm{O}$ 1s profile at 531.8 and $532.2 \mathrm{eV}$ could be attributed to $\mathrm{C}=\mathrm{O}$ and C-OH/C-O-C, and the weak shoulders at 531.0 and $534.1 \mathrm{eV}$ are associated with quinone/pyridine and carboxylic O. ${ }^{20,35}$ It is well known that ketone groups, carboxylic groups, and cyclic oxygen show a stronger anchoring effect towards lithium sulfides. ${ }^{34}$ The S $2 p$ XPS spectrum display two major peaks at 165.0 and $163.8 \mathrm{eV}$, corresponding to the spin-orbit coupling of $\mathrm{S} 2 \mathrm{p}_{3 / 2}$ and $2 \mathrm{p}_{1 / 2}$. The weak peaks at 165.7 and $164.5 \mathrm{eV}$ are assigned to S-O species, while the small broad peak at $168.4 \mathrm{eV}$ is ascribed to sulfate due to the oxidation of sulfur in air. ${ }^{36}$ According to the EDS spectra analysis, the doping concentration of N, O atoms is 4.2 at\% and 7.8 at\% in NOCMs, respectively (Figure S7). The XPS peaks for N, O further confirm the successfully incorporation of $\mathrm{N}, \mathrm{O}$ atoms into the carbon lattice, promising strong anchoring sites for polysulfides and resulting in better cycling stability.

(a)
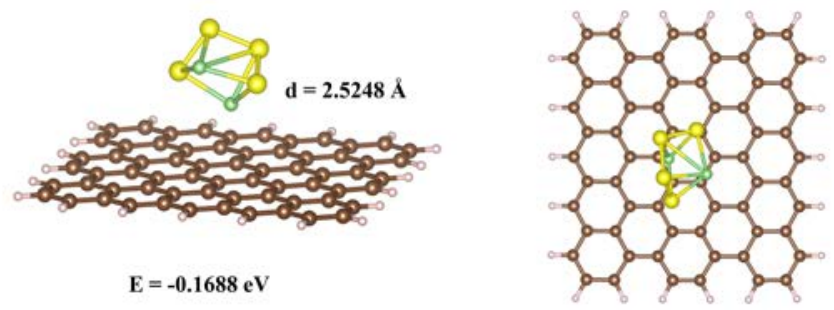

(b)
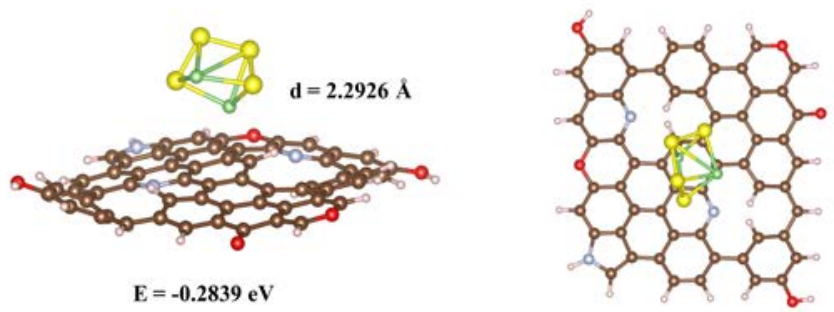

Figure 6. Schematic illustration of the structure, adsorption energy, and distance of $\mathrm{Li}_{2} \mathrm{~S}_{4}$ from (a) a pristine carbon surface and (b) an N, O co-doped carbon surface. The yellow, green, brown, red, light blue, and light pink balls are S, Li, C, O, N, and $\mathrm{H}$ atoms, respectively. 
To better understand the superiority of $\mathrm{N}$, O co-doped carbon towards preventing the polysulfide shuttle effect, the adsorption energies of $\mathrm{Li}_{2} \mathrm{~S}_{4}$ on carbon surfaces were studied by density functional theory (DFT) calculations (Figure 6). The pores in the NOCMs sample seem to be assembled from a few layers of graphene, and therefore, the single layer graphene model was adopted along with the intermediate product $\mathrm{Li}_{2} \mathrm{~S}_{4}$. Generally, negative adsorption energy suggests a stable adsorption configuration, and the adsorption stability increases as its absolute value increases. The calculation results show that the adsorption energy of $\mathrm{Li}_{2} \mathrm{~S}_{4} \mathrm{is}-0.168 \mathrm{eV}$ and $-0.283 \mathrm{eV}$ for pure carbon and $\mathrm{N}, \mathrm{O}$ co-doped carbon, respectively. This indicates that doping with $\mathrm{N}, \mathrm{O}$ atoms could significantly enhance the interaction between $\mathrm{Li}_{2} \mathrm{~S}_{4}$ and the carbon host, which is beneficial to the activity of the cell and effectively prevents shuttling of polysulfides. Otherwise, the distance between $\mathrm{Li}_{2} \mathrm{~S}_{4}$ and the carbon host is decreased after $\mathrm{N}$, O co-doping (2.2926 $\AA$ for N, O co-doped carbon surface and $2.5248 \AA$ for pristine carbon surface), which also demonstrates the enhanced interaction between the molecule and the surface. 

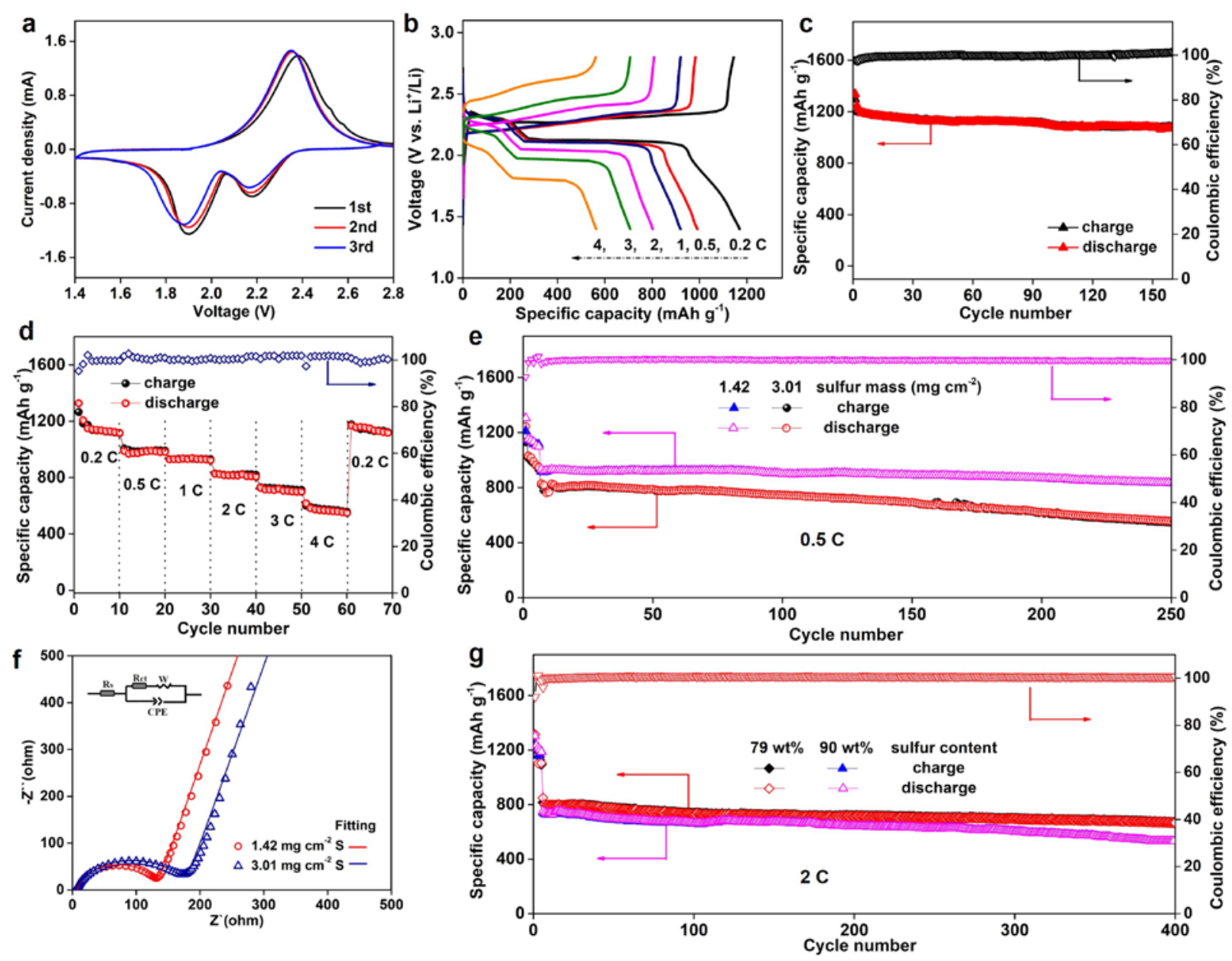

Figure 7. (a) CV curves for the first 3 cycles of the S@NOCMs (79 wt\%) composite electrode at a sweep rate of $0.1 \mathrm{mV} \mathrm{s}^{-1}$. (b) Charge-discharge profiles of the S@NOCMs composite at various current rates. (c) Cycling performance of S@NOCMs at the 0.2 C rate (1 C = 1672 mAh g$\left.{ }^{-1}\right)$. (d) Rate performance of S@NOCMs composite electrode. The areal sulfur mass loading was around $1.2 \mathrm{mg} \mathrm{cm}^{-2}$ for the (b-d) graphs. (e) Cycling performances of the electrodes based on different sulfur loading at the $0.5 \mathrm{C}$ rate, and (f) the corresponding Nyquist plots, with the equivalent circuit in the inset. (g) Cycling performances of S@NOCMs composites with different sulfur contents of $79 \mathrm{wt} \%$ and $90 \mathrm{wt} \%$ at the $2 \mathrm{C}$ rate. For (e, g), the electrochemical performance was tested with an activation process for the first 5 cycles at the $0.1 \mathrm{C}$ rate.

Figure 7a displays the cyclic voltammetry (CV) curves of the S@NOCMs composite, recorded at a scan of $0.1 \mathrm{mV} \mathrm{s}^{-1}$ in the potential range of $1.4-2.8 \mathrm{~V}$ (vs. $\mathrm{Li}^{-} / \mathrm{Li}^{+}$). In the first cycle, 
two reduction peaks centered at $1.92 \mathrm{~V}$ and $2.21 \mathrm{~V}$ are observed. The peak at $2.21 \mathrm{~V}$ is related to the lithiation of orthorhombic $\mathrm{S}_{8}$ to the high-order lithium polysulfides $\operatorname{Li}_{2} \mathrm{~S}_{x}(6<x \leq 8)$. The peak located at $1.91 \mathrm{~V}$ is associated with the further reduction of high-order lithium polysulfides to the low-order lithium polysulfides $\mathrm{Li}_{2} \mathrm{~S}_{x}(2 \leq x \leq 6)$, ending with $\mathrm{Li}_{2} \mathrm{~S}$. ${ }^{4,} 37$ Two corresponding oxidation peaks are overlapped and formed one broad peak at about $2.5-2.7 \mathrm{~V}$, which corresponds to the multistep conversion of $\mathrm{Li}_{2} \mathrm{~S}$ to intermediate lithium polysulfides and eventually to $\mathrm{S}_{8}{ }^{4,37}$ In the following cycles, the shapes of the CV curves are very similar to that of the first cycle, with hardly any change in the positions, indicating that the S@NOCMs composite cathode has good reversibility. Figure 7b presents the discharge-charge profiles of S@NOCMs at various rates. Two obvious discharging platforms and one charging platform are exhibited in every stage of the rates, agreeing well with the CV profiles. At low current densities, the charge-discharge profiles show flat and long plateaus as well as low polarization, which implies that the sample will have good electrochemical performance at this level. More importantly, the plateaus could be well maintained at higher rates, suggesting good reaction kinetics, and may be expected to correspond to good rate capacity. ${ }^{31,23}$

The cycling performance of S@NOCMs electrodes was tested at the $0.2 \mathrm{C}$ rate within the potential window of 1.4-2.8 V, as is shown in Figure 7c. The initial discharging capacity of the S@NOCMs composite is as high as $1327 \mathrm{mAh} \mathrm{g}^{-1}$, corresponding to a high sulfur utilization of $79 \%$, based on the theoretical capacity of sulfur (1672 $\mathrm{mAh} \mathrm{g}^{-1}$ ). The discharging capacity rapidly dropped to $1233 \mathrm{mAh} \mathrm{g}^{-1}$ at the $2^{\text {nd }}$ cycle, because the electrolyte additive $\mathrm{LiNO}_{3}$ is reduced irreversibly on the carbon surface, and there are some side reactions. ${ }^{39}$ By benefiting from the N, O-dual doping and the good physical and chemical confinement of sulfur, the capacity of S@NOCMs electrode was maintained at 1071 mAh g-1 after 160 cycles with nearly 
$100 \%$ Coulombic efficiency, corresponding to capacity retention of $80.7 \%$ of the initial value and only $0.082 \%$ capacity decay per cycle (starting from the second circle). The rate capability of S@NOCMs electrode was evaluated at various current densities, ranging from $0.2 \mathrm{C}$ to $4 \mathrm{C}$, as is shown in Figure 7d. The average discharge capacities for S@NOCMs electrode are 1125, 985, 923, 810, 701, and $558 \mathrm{mAh} \mathrm{g}^{-1}$ at corresponding rates of $0.2,0.5,1,2,3$, and $4 \mathrm{C}$, respectively. When the current density was returned to the $0.2 \mathrm{C}$ rate, the discharge capacity could be recovered to $1122 \mathrm{mAh} \mathrm{g}^{-1}$, indicating the good stability and outstanding rate performance of this electrode material. Compared to the previous studies, ${ }^{8,11,29,30}$ the S@NOCMs composite with high sulfur content of 79\% displayed improved high rate capacities and good cycling stability. The high sulfur utilization and excellent rate performance are attributed to the ultrafine sulfur nanoparticles confined in the highly conductive $\mathrm{N}$, O dual-doped porous carbon framework, in which the stronger interfacial interaction between the carbon host and the polysulfide guests effectively prevents the shuttle effect.

High areal sulfur loading is a key parameter for achieving high energy density, and is essential for practical applications of Li-S batteries. Therefore, the cycling performances of S@NOCMs composites with areal sulfur loading of $1.42 \mathrm{mg} \mathrm{cm}^{-2}$ and $3.01 \mathrm{mg} \mathrm{cm}^{-2}$ were tested at the $0.5 \mathrm{C}$ rate after an activation process for the first 5 cycles. Both composites show high capacity and stable long-term cycling stability. The electrode with $1.42 \mathrm{mg} \mathrm{cm}^{-2}$ sulfur loading presents $813 \mathrm{mAh} \mathrm{g}^{-1}$ after 250 cycles, corresponding to good cycling stability with $88 \%$ capacity retention (923 $\mathrm{mAh} \mathrm{g}^{-1}$ at the $6^{\text {th }}$ cycle) and $0.05 \%$ capacity decay per cycle. The electrode with $3.01 \mathrm{mg} \mathrm{cm}^{-2}$ sulfur loading still displays $564 \mathrm{mAh} \mathrm{g}^{-1}$ after 250 cycles, corresponding to good cycle stability with $74 \%$ capacity retention (762 $\mathrm{mAh} \mathrm{g}^{-1}$ at the $6^{\text {th }}$ cycle) and $0.10 \%$ capacity decay per cycle. The electrode with $4.4 \mathrm{mg} \mathrm{cm}^{-2}$ sulfur loading displays 367 
$\mathrm{mAh} \mathrm{g}^{-1}$ after 130 cycles, corresponding to $74 \%$ capacity retention (569 $\mathrm{mAh} \mathrm{g}^{-1}$ at the $1^{\text {st }}$ cycle) and $0.27 \%$ capacity decay per cycle (Figure S8). The performance of the electrode with high sulfur loading and relatively lower capacity was confirmed by electrochemical impedance spectroscopy (EIS). Figure 7f shows the Nyquist plots of S@NOCMs samples with different sulfur loading at the same voltage of $2.2 \mathrm{~V}$ during the charging process after 10 cycles, and the inset displays the equivalent circuit. The low-frequency region reflects the Warburg impedance (solid-state diffusion of $\mathrm{Li}$ ions in the active materials), the intercept on the $\mathrm{Z}^{\prime}$ axis is related to electrolyte resistance $\left(R_{\mathrm{e}}\right)$, and the semicircle in the high-frequency region corresponds to charge transfer resistance $\left(R_{\mathrm{ct}}\right)$ at the electrode-electrolyte interface. ${ }^{11,40}$ The $R_{\mathrm{ct}}$ was determined to be $123 \Omega$ for loading of $1.42 \mathrm{mg} \mathrm{cm}^{-2}$ and $192 \Omega$ for $3.01 \mathrm{mg} \mathrm{cm}^{-2}$, indicating that the capacity loss may be because the increasing thickness of the active material leads to decreasing interface between the current collector, the electrolyte, and the active material. ${ }^{11,40}$

The cycling performances of S@NOCMs composites with different sulfur loading of 79 wt\% and $90 \mathrm{wt} \%$ (Figure $7 \mathrm{~g}$ ) were investigated at the high current density of $2 \mathrm{C}$. They both show similar specific capacity and long-term cycling stability, as well as confirming the high sulfur utilization. The S@NOCMs composite with 79 wt\% sulfur maintained a high specific capacity of $667 \mathrm{mAh} \mathrm{g}^{-1}$ after 400 cycles. The S@NOCMs electrode with 90 wt $\%$ sulfur maintained a high capacity of $602 \mathrm{mAh} \mathrm{g}^{-1}$ after 300 cycles, and $533 \mathrm{mAh} \mathrm{g}^{-1}$ at the $400^{\text {th }}$ cycle. These results demonstrate the excellent cycling stability at a high current density of S@NOCMs composites and confirm that the polysulfide shuttle effect was efficiently suppressed. First, the porous host material NOCMs could improve the electrical conductivity of the cathode composites, while the micro/mesopores facilitate rapid access of the electrolyte to the electrode interior, contributing to the high capacity. Furthermore, the unique porous structure can also 
provide more active sites for sulfur absorption and mitigate the diffusion of polysulfide, reducing the loss of active materials. More importantly, the $\mathrm{N}$, $\mathrm{O}$ functional groups on the surfaces of NOCMs can enhance the adsorption through chemical bonding between NOCMs and sulfur/polysulfides, improving the coulombic efficiency and cycling stability. ${ }^{18,20}$ In addition, the electrolyte additive $\mathrm{LiNO}_{3}$ could also help to inhibit the dissolution of polysulfides by the formation the of a stable passivation film on the surface of Li metal. ${ }^{7}$ As a result, the S@NOCMs composite with high specific capacity and long cycling stability is a promising cathode for Li-S batteries.

\section{Conclusion}

In summary, $\mathrm{N}$, O dual doped highly porous carbon microrods with large pore volume, high surface area, and micro/mesopores have been successfully synthesized via a simple calcination and acid treament process. The large pore volume and high surface area permit high sulfur loading in the amount of $79-90 \mathrm{wt} \%$ in the composite and contribute to the physical anchoring effect for sulfur or polysulfides. The porous carbon microrods codoped with $\mathrm{N}$, O atoms signifantly enhance the chemical anchoring effect of sulfur/polysulfides to the carbon host via dipole-dipole electrostatic interaction, guaranting high sulfur utilization and preventing shuttling of polysulfides. Thus, the S@NOCMs composite delivers excellent rate performance and longterm cycling stability, so that it could maintain $813 \mathrm{mAh} \mathrm{g}^{-1}$ after 250 cycles at the $0.5 \mathrm{C}$ rate and $667 \mathrm{mAh} \mathrm{g}^{-1}$ after 400 cycles at the high $2 \mathrm{C}$ rate. Such superior electrochemical performance of the S@NOCMs composite can pave the way to the construction of high-energy commercially viable Li-S batteries.

\section{Supporting information}


TEM and SEM images of Ni(DMG) $)_{2}$ microrods; XRD pattern and TEM images of Ni@NCMs. SEM images of Ni@NCMs composite. XRD pattern and TGA curve of S@NOCMs composite with $90 \mathrm{wt} \%$ sulfur. These materials are available free of charge via the Internet at http://pubs.acs.org.

\section{Corresponding Author}

Professor Shixue Dou, E-mail: shi_dou@uow.edu.au;

Dr. Zhongchao Bai, E-mail: baizhongchao@tyut.edu.au;

\section{Author Contributions}

The manuscript was written through contributions of all authors. All authors have given approval to the final version of the manuscript.

\section{Acknowledgements}

This work is supported by the Australian Research Council (ARC) through a Discovery Projects (DP160102627) and a Linkage Project (LP160100273), National Natural Science Foundation of China (No. 51671140), a Research Project Supported by the Shanxi Scholarship Council of China (No. 2015-034), the Natural Science Foundation of Shanxi Province of China (201701D221077), Science and Technology Innovation Project of Shanxi Higher School (No. 2017129), the authors thank Dr.Tania Silver for her helpful discussions.

\section{References:}

[1] Tarascon, J.-M.; Armand, M. Issues and challenges facing rechargeable lithium batteries. Nature 2001, 414, 359-367.

[2] Goodenough, J. B.; Kim, Y. Challenges for rechargeable Li batteries. Chem. Mater. 2010, 22, 587-603.

[3] Evers, S.; Nazar, L. F. New approaches for high energy density lithium-sulfur battery cathodes. Acc. Chem. Res. 2013, 46, 1135-1143. 
[4] Manthiram, A.; Fu, Y.; Chung, S.-H.; Zu, C.; Su, Y.-S. Rechargeable lithium-sulfur batteries. Chem. Rev. 2014, 114, 11751-11787.

[5] Li, Z.; Yuan, L.; Yi, Z.; Sun, Y.; Liu, Y.; Jiang, Y.; Shen, Y.; Xin, Y.; Zhang, Z.; Huang, Y. Insight into the electrode mechanism in lithium-sulfur batteries with ordered microporous carbon confined sulfur as the cathode. Adv. Energy Mater. 2014, 4, 1301473-1301480.

[6] Wild, M.; O’Neill, L.; Zhang, T.; Purkayastha, R.; Minton, G.; Marinescu, M.; Offer, G. J. Lithium sulfur batteries, a mechanistic review. Energy Environ. Sci. 2015, 8, 3477-3494.

[7] Seh, Z. W.; Sun, Y.; Zhang, Q.; Cui, Y. Designing high-energy lithium-sulfur batteries. Chem. Soc. Rev. 2016, 45, 5605-5634.

[8] Fu, Y.; Su, Y.-S.; Manthiram, A. Sulfur-carbon nanocomposite cathodes improved by an amphiphilic block copolymer for high-rate lithium-sulfur batteries. ACS Appl. Mater. Interfaces 2012, 4, 6046-6052.

[9] Liang, X.; Nazar, L. F. In Situ Reactive assembly of scalable core-shell sulfur- $\mathrm{MnO}_{2}$ composite cathodes. ACS Nano 2016, 10, 4192-4198.

[10] Seh, Z. W.; Yu, J. H.; Li, W.; Hsu, P.-C.; Wang, H.; Sun, Y.; Yao, H.; Zhang, Q.; Cui, Y. Two-dimensional layered transition metal disulphides for effective encapsulation of highcapacity lithium sulphide cathodes. Nat. Commun. 2014, 5, 5017-5024.

[11] Zhou, J.; Li, R.; Fang, X.; Chen, Y.; Han, R.; Li, W.; Zheng, J.; Wang, B.; Li, X. Rational design of a metal-organic framework host for sulfur storage in fast, long-cycle Li-S batteries. Energy Environ. Sci. 2014, 7, 2715-2724.

[12] Ye, H.; Yin, Y.-X.; Xin, S.; Guo, Y.-G. Tuning the porous structure of carbon hosts for loading sulfur toward long lifespan cathode materials for Li-S batteries. J. Mater. Chem. A 2013, 1, 6602-6608. 
[13] Ye, H.; Yin, Y.-X.; Guo, Y.-G. Insight into the loading temperature of sulfur on sulfur/carbon cathode in lithium-sulfur batteries. Electrochim.Acta 2015, 185, 62-68.

[14] You, Y.; Zeng, W.; Yin, Y.-X.; Zhang, J.; Yang, C.-P.; Zhu, Y.; Guo, Y.-G. Hierarchically micro/mesoporous activated graphene with a large surface area for high sulphur loading in Li-S batteries. J. Mater. Chem. A 2015, 3, 4799-4802.

[15] Yang, C.-P.; Yin, Y.-X.; Guo, Y.-G.; Wan, L.-J. Electrochemical (de)Lithiation of 1D sulfur chains in Li-S batteries: A Model System Study. J. Am. Chem. Soc. 2015, 137, 2215-2218. [16] Du, W.-C.; Yin, Y.-X.; Zeng, X.-X.; Shi, J.-L.; Zhang, S.-F.; Wan, L.-J.; Guo, Y.-G. Wet chemistry synthesis of multidimensional nanocarbon-sulfur hybrid materials with ultrahigh sulfur loading for lithium-sulfur batteries. ACS Appl. Mater. Interfaces 2016, 8, 3584-3590.

[17] Zhang, J.; Yang, C.-P.; Yin, Y.-X.; Wan, L.-J.; Guo, Y.-G. Sulfur encapsulated in graphitic carbon nanocages for high-rate and long-cycle lithium-sulfur batteries. Adv. Mater. 2016, 28, 9539-9544.

[18] Sahore, R.; Levin, B. D. A.; Pan, M.; Muller, D. A.; DiSalvo, F. J.; Giannelis, E. P. Design principles for optimum performance of porous carbons in lithium-sulfur batteries. Adv. Energy Mater. 2016, 6, 1600134-1600142.

[19] Zheng, G.; Zhang, Q.; Cha, J. J.; Yang, Y.; Li, W.; Seh, Z. W.; Cui, Y. Amphiphilic surface modification of hollow carbon nanofibers for improved cycle life of lithium sulfur batteries, Nano Lett. 2013, 13, 1265-1270.

[20] Hou, T.-Z.; Chen, X.; Peng, H.-J.; Huang, J.-Q.; Li, B.-Q.; Zhang, Q.; Li, B. Design principles for heteroatom-doped nanocarbon to achieve strong anchoring of polysulfides for lithium-sulfur batteries. Small 2016, 12, 3283-3291. 
[21] Ji, L.; Rao, M.; Zheng, H.; Zhang, L.; Li, Y.; Duan, W.; Guo, J.; Cairns, E. J.; Zhang, Y. Graphene oxide as a sulfur immobilizer in high performance lithium/sulfur cells. J. Am. Chem. Soc. 2011, 133, 18522-18525.

[22] Song, J.; Gordin, M. L.; Xu, T.; Chen, S.; Yu, Z.; Sohn, H.; Lu, J.; Ren, Y.; Duan, Y.; Wang, D. Strong lithium polysulfide chemisorption on electroactive sites of nitrogen-doped carbon composites for high-performance lithium-sulfur battery cathodes. Angew. Chem. Int. Ed. 2015, $54,4325-4329$.

[23] Zhou, G.; Paek, E.; Hwang, G. S.; Manthiram, A. Long-life Li/polysulphide batteries with high sulphur loading enabled by lightweight three-dimensional nitrogen/sulphur-codoped graphene sponge. Nat. Commun. 2015, 6, 7760-7770.

[24] Yuan, S.; Bao, J. L.; Wang, L.; Xia, Y.; Truhlar, D. G.; Wang, Y. Graphene-supported nitrogen and boron rich carbon layer for improved performance of lithium-sulfur batteries due to enhanced chemisorption of lithium polysulfides. Adv. Energy Mater. 2016, 6, 1501733-1501791. [25] Wang, N.; Chen, L.; Ma, X.; Yue, J.; Niu, F.; Xu, H.; Yang, J.; Qian, Y. Facile synthesis of hierarchically porous $\mathrm{NiO}$ microtubes as advanced anode materials for lithium-ion batteries. $J$. Mater. Chem. A 2014, 2, 16847-16850.

[26] Gómez-Serrano, V.; Acedo-Ramos, M.; López-Peinado, A. J.; Valenzuela-Calahorro, C. Oxidation of activated carbon by hydrogen peroxide. Study of surface functional groups by FTi.r. Fuel 1994, 73, 387-395.

[27] Moreno-Castilla, C.; Ferro-García, M. A.; Joly, J. P.; Bautista-Toledo, I.; Carrasco-Marín, F.; Rivera-Utrilla, J. Activated carbon surface modifications by nitric acid, hydrogen peroxide, and ammonium peroxydisulfate treatments. Langmuir 1996, 11, 4386-4392. 
[28] Bhatnagar, A.; Hogland, W.; Marques, M.; Sillanpää, M. An overview of the modification methods of activated carbon for its water treatment applications. Chem. Eng. J. 2013, 219, 499511.

[29] Zhang, C.; Fu, L.; Liu, N.; Liu, M.; Wang, Y.; Liu, Z. Synthesis of Nitrogen-Doped Graphene Using Embedded Carbon and Nitrogen Sources. Adv. Mater. 2011, 23, 1020-1024.

[30] Mi, K.; Chen, S.; Xi, B.; Kai, S.; Jiang, Y.; Feng, J.; Qian, Y.; Xiong, S. Sole chemical confinement of polysulfides on nonporous nitrogen/oxygen dual-doped carbon at the kilogram scale for lithium-sulfur batteries. Adv. Funct. Mater. 2017, 27, 1604265-1604277.

[31] Li, G.; Sun, J.; Hou, W.; Jiang, S.; Huang, Y.; Geng, J. Three-dimensional porous carbon composites containing high sulfur nanoparticle content for high-performance lithium-sulfur batteries. Nat. Commun. 2016, 7, 10601-10610.

[32] Elazari, R.; Salitra, G.; Garsuch, A.; Panchenko, A.; Aurbach, D. Sulfur-impregnated activated carbon fiber cloth as a binder-free cathode for rechargeable Li-S batteries. Adv. Mater. 2011, 23, 5641-5644.

[33] Kruk, M.; Jaroniec, M. Gas adsorption characterization of ordered organic-inorganic nanocomposite materials. Chem. Mater. 2001, 13, 3169-3183.

[34] Liu, J.; Li, W.; Duan, L.; Li, X.; Ji, L.; Geng, Z.; Huang, K.; Lu, L.; Zhou, L.; Liu, Z.; Chen, W.; Liu, L.; Feng, S.; Gang, Y. A graphene-like oxygenated carbon nitride material for improved cycle-life lithium/sulfur batteries. Nano Lett. 2015, 15, 5137-5142.

[35] Yuan, S.; Guo, Z.; Wang, L.; Hu, S.; Wang, Y.; Xia, Y. Leaf-like graphene-oxide-wrapped sulfur for high performance lithium-sulfur battery. Adv. Sci. 2015, 2, 1500071-1500080. 
[36] Zhou, G.; Yin, L.-C.; Wang, D.-W.; Li, L.; Pei, S.; Gentle, I. R.; Li, F.; Cheng, H.-M. Fibrous hybrid of graphene and sulfur nanocrystals for high-performance lithium sulfur batteries. ACS Nano 2013, 7, 5367-5375.

[37] Bruce, P. G.; Freunberger, S. A.; Hardwick, L. J.; Tarascon, J.-M. Li-O $\mathrm{O}_{2}$ and Li-S batteries with high energy storage. Nat. Mater. 2012, 11, 19-29.

[38] Schuster, J.; He, G.; Mandlmeier, B.; Yim, T.; Lee, K. T.; Bein, T.; Nazar, L. F. Spherical ordered mesoporous carbon nanoparticles with high porosity for lithium-sulfur batteries. Angew. Chem. 2012, 124, 3651-3655

[39] Zhang, S. S. Role of $\mathrm{LiNO}_{3}$ in rechargeable lithium/sulfur battery. Electrochim. Acta 2012, 70, 344-348.

[40] Wang, C.; Wan, W.; Huang, Y.; Chen, J.; Zhou, H. H.; Zhang, X. X. Hierarchical MoS 2 nanosheet/active carbon fiber cloth as a binder-free and free-standing anode for lithium-ion batteries. Nanoscale 2014, 6, 5351-5358. 


\section{Table of contents}

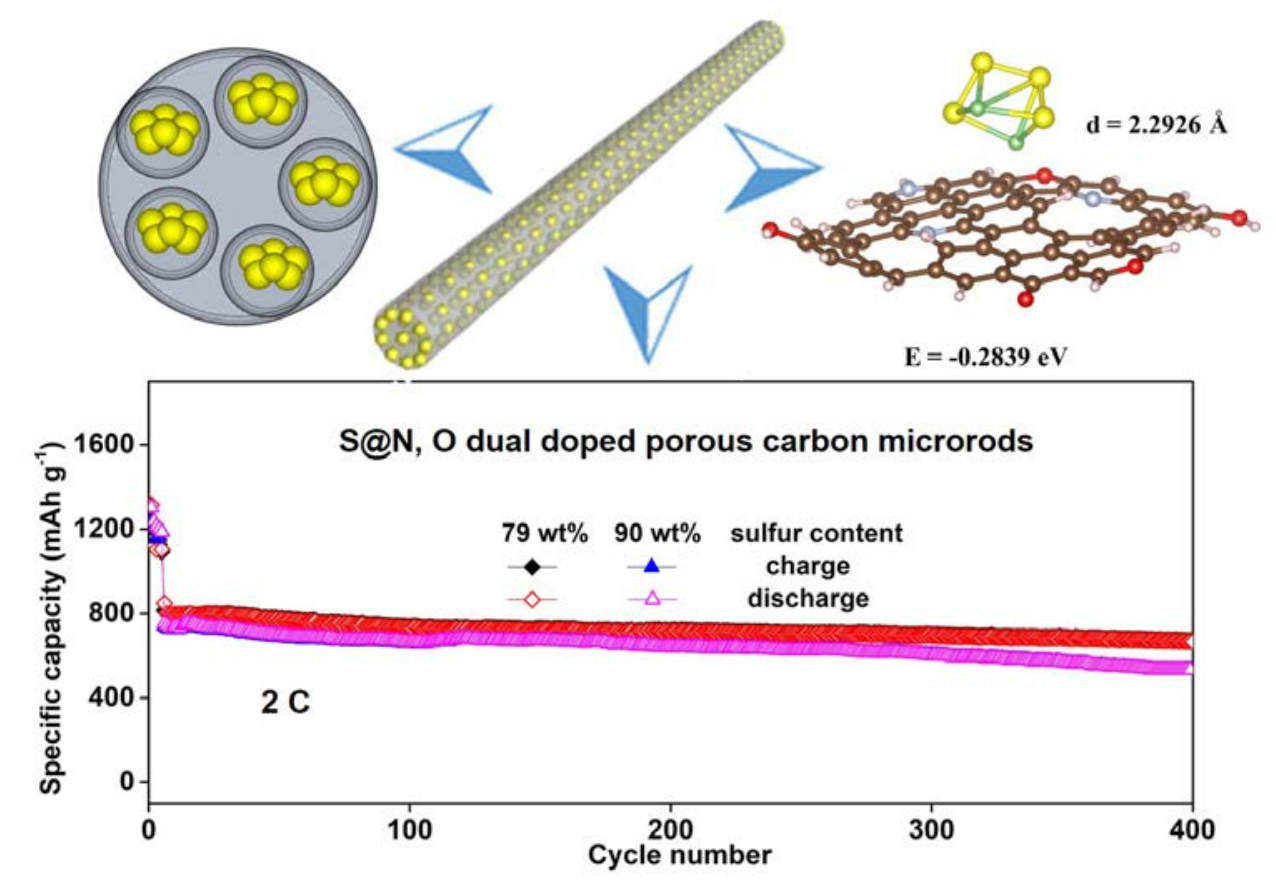




\section{Supporting Information}

Synergistically Enhanced Interfacial Interaction to Polysulfide via N,O Dual-Doped Highly Porous Carbon Microrods for Advanced Lithium-Sulfur Batteries

Nana Wang, ${ }^{a b}$ Zhongfei Xu, ${ }^{a}$ Xun Xu, ${ }^{a}$ Ting Liao, ${ }^{c}$ Bin Tang, ${ }^{b}$ Zhongchao Bai, $*{ }^{a b}$ and Shixue $\mathrm{Dou}^{*^{a}}$

a Institute for Superconducting and Electronic Materials, Australian Institute of Innovative Materials, University of Wollongong, Innovation Campus, Squires Way, North Wollongong, New South Wales 2500, Australia,

${ }^{b}$ College of Materials Science and Engineering, Taiyuan University of Technology, Taiyuan, Shanxi 030024, PRC,

${ }^{c}$ School of Chemistry, Physics and Mechanical Engineering, Queensland University of Technology, QLD 4000, Australia.

Corresponding authors’email: shi@uow.edu.au; Baizhongchao@tyut.edu.au 

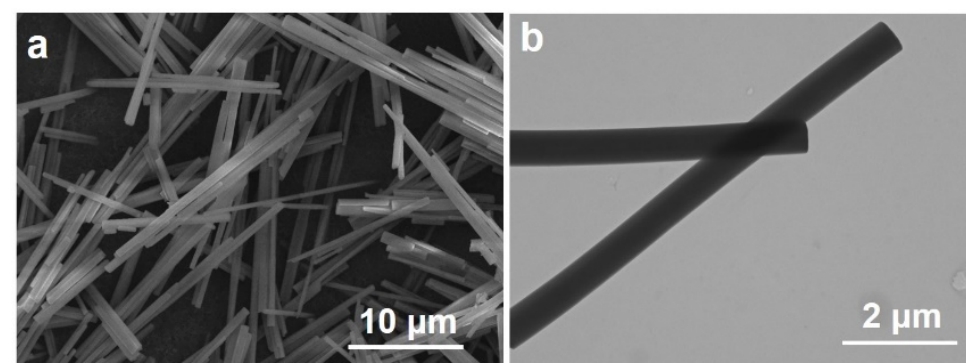

Figure S1. (a) SEM and (b) TEM images of Ni(DMG) $)_{2}$ microrods.
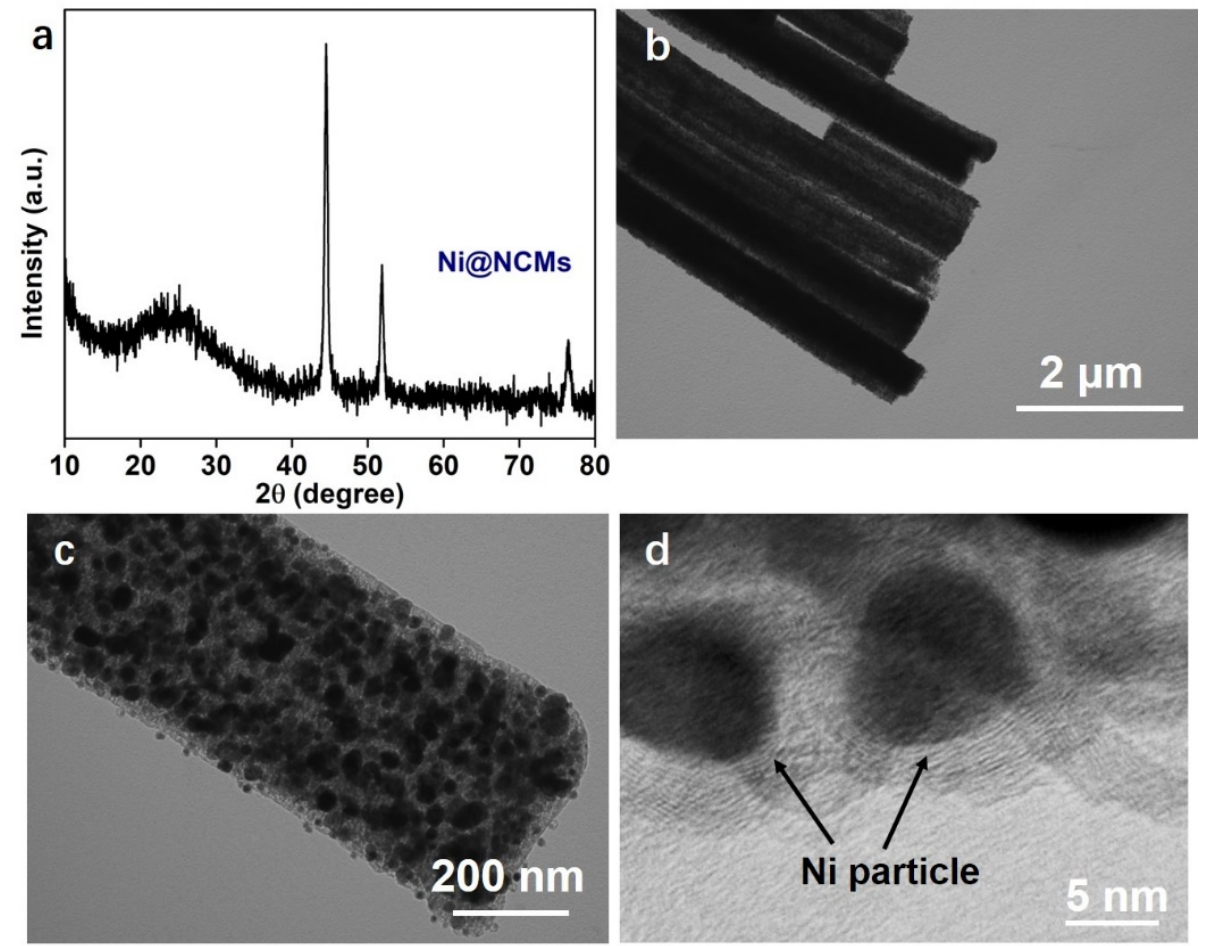

Figure S2. (a) XRD pattern, and (b) low magnification and (c, d) high magnification TEM images of Ni@NCMs.
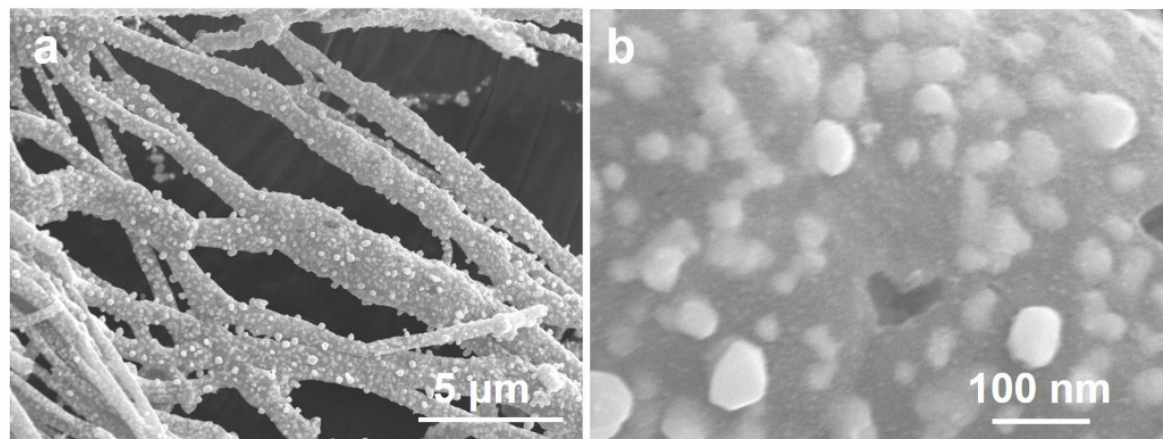

Figure S3. (a, b) SEM images of Ni@NCMs composite at different magnifications. 


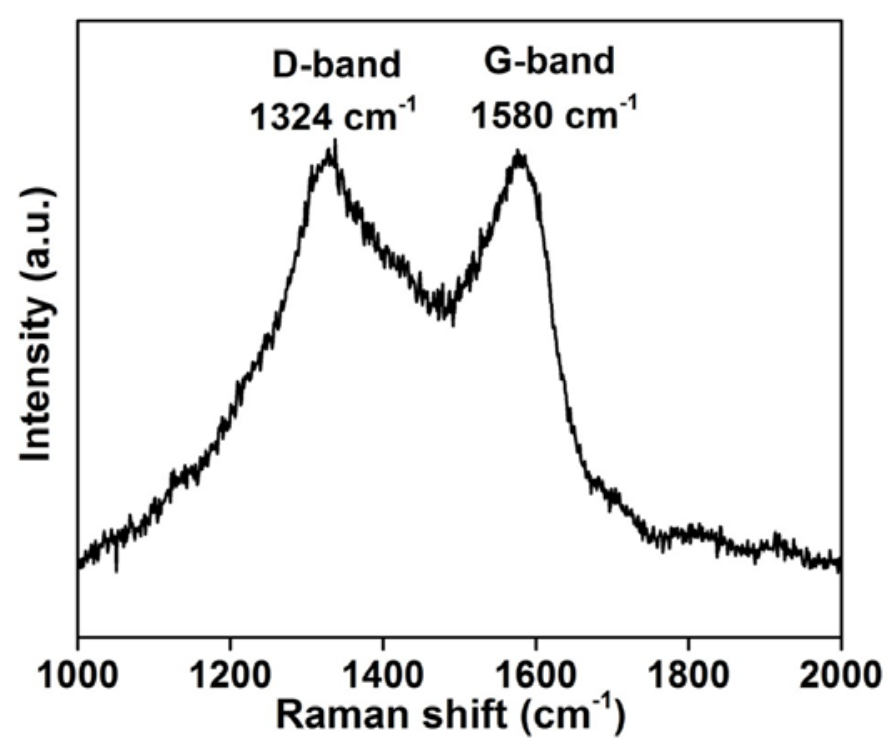

Figure S4. Raman spectrum of NOCMs sample.
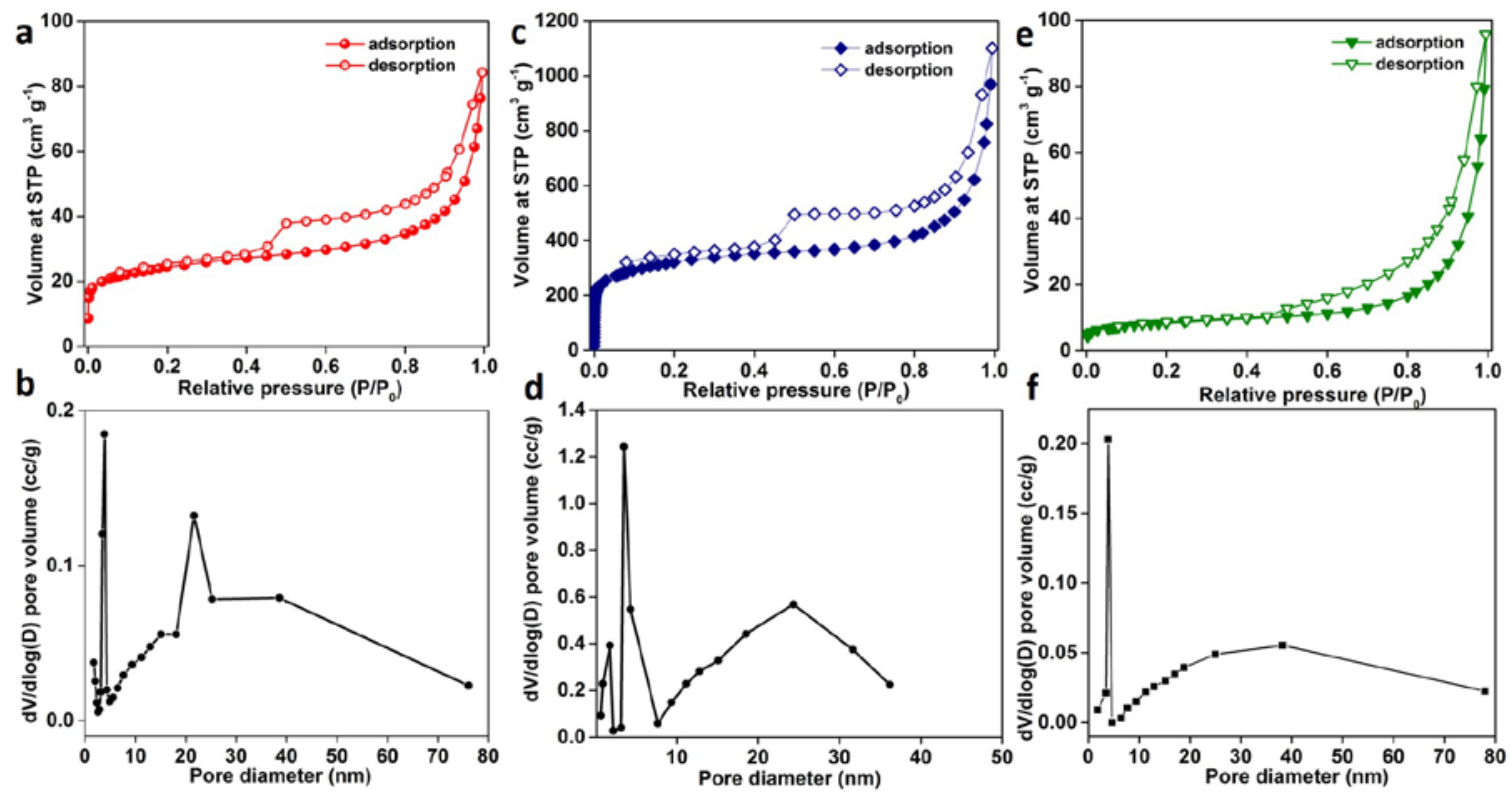

Figure S5. Nitrogen adsorption/desorption isotherms and corresponding pore size distribution of (a, b) Ni@NCMs, (c, d) NOCMs, and (e, f) S@NOCMs (79 wt. \% S) composite.
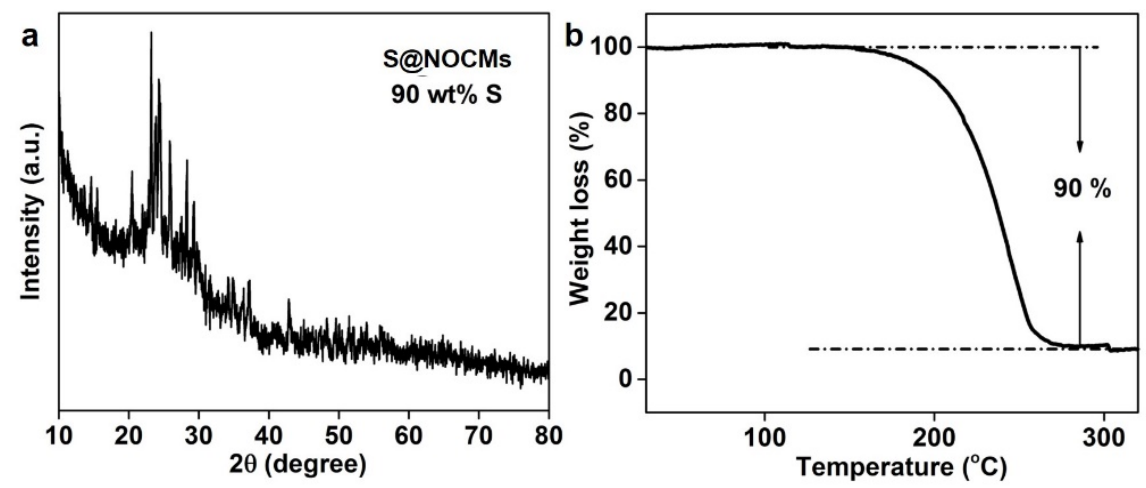

Figure S6. (a) XRD pattern and (b) TGA curve of S@NOCMs composite with 90 wt\% sulfur. 
a

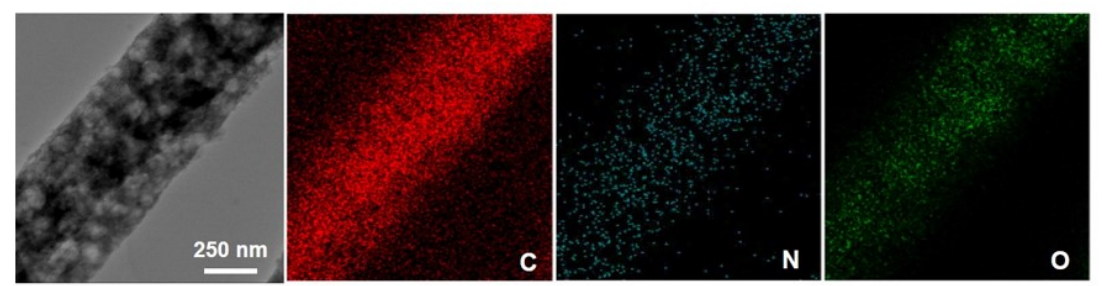

b

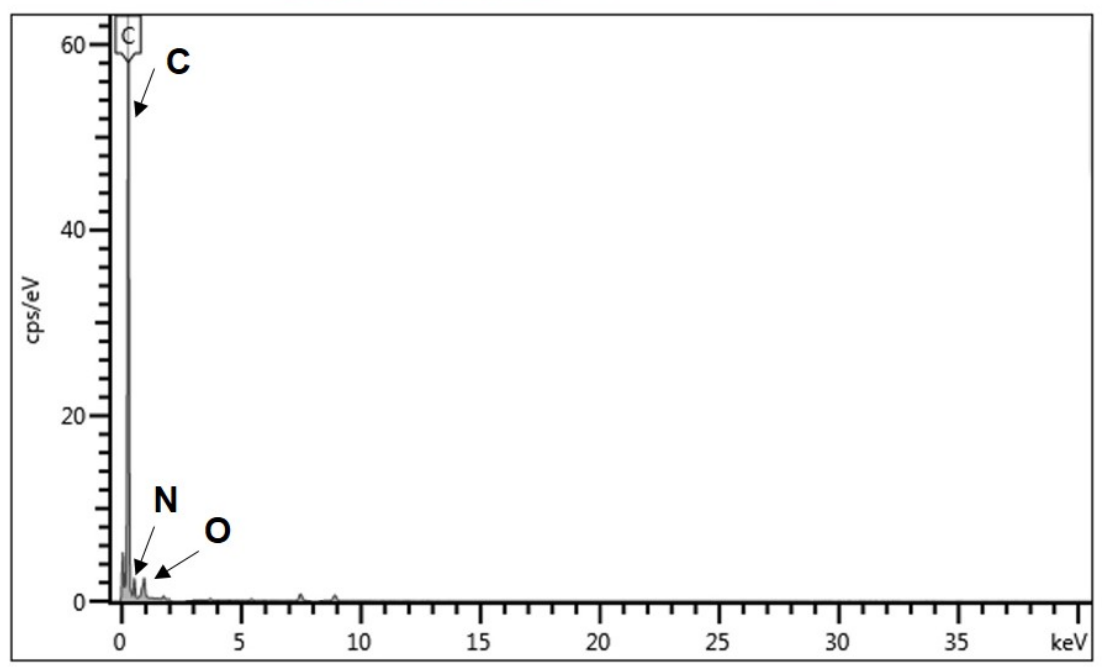

Figure S7. (a) Corresponding carbon, nitrogen and oxygen elemental mappings of NOCMs. (b) EDS spectrum of NOCMs.

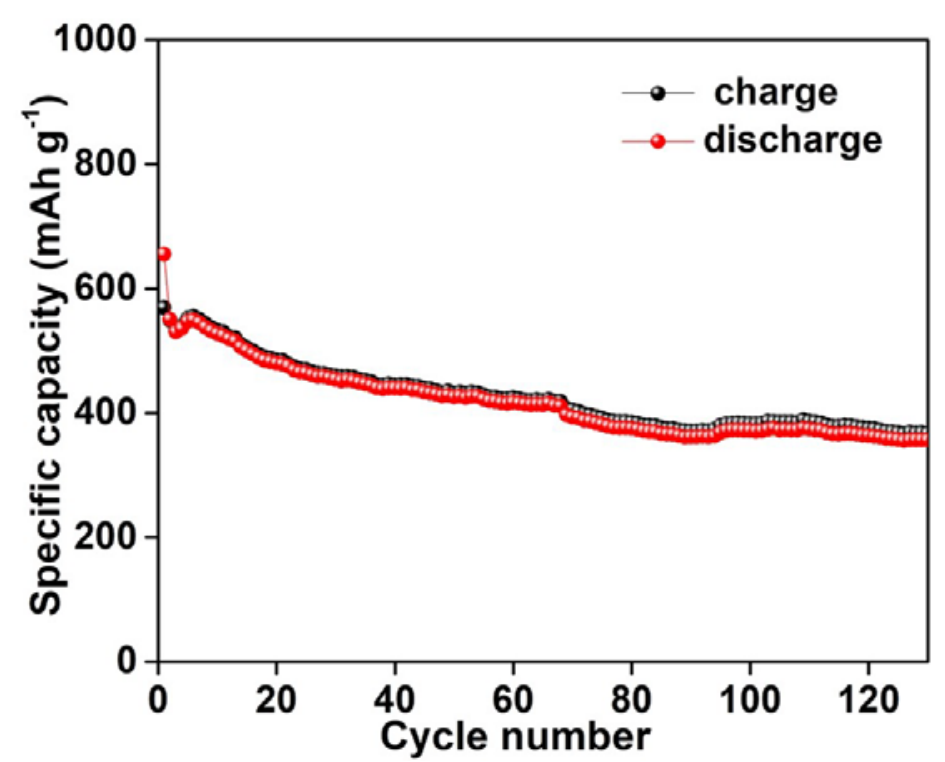

Figure S8. Cycling performances of S@NOCMs (79 wt. \%) with areal sulfur mass loading was around $4.4 \mathrm{mg} \mathrm{cm}^{-2}$. 